Copyright@2004 Springer Science+Business Media B.V. Reprinted with permission from:

T. Roman, M. Enescu and V. Koivunen. Joint time-domain tracking of channel and frequency offsets for MIMO OFDM systems. In Wireless Personal Communications, Kluwer, Vol. 31, Nos 3-4, pp. 181-200, December 2004.

DOI: $10.1007 / \mathrm{s} 11277-004-3826-6$

The original publication is available at www.springerlink.com 


\title{
Joint Time-Domain Tracking of Channel and Frequency Offsets for MIMO OFDM Systems
}

\author{
TIMO ROMAN, MIHAI ENESCU and VISA KOIVUNEN \\ Signal Processing Laboratory, Helsinki University of Technology, P.O. Box 3000, FIN-02015 HUT, Finland \\ E-mail:timo.roman@hut.fi,mihai.enescu@hut.fi,visa.koivunen@hut.fi
}

\begin{abstract}
In this paper we address the problem of joint channel and frequency offset estimation and tracking in multiple-input multiple-output (MIMO) OFDM systems for mobile users. The proposed method stems from extended Kalman filtering and is suitable for time-frequency-space selective channels. Separate offset for each MIMO channel branch is considered because of the mobility and rich scattering. The channel taps and the frequency offsets are estimated in time-domain while the equalization is performed in frequency domain. Simulation results demonstrate that the proposed method tracks time-varying channels and frequency offsets with high fidelity. Realistic channel models are used in mobile scenarios. The proposed time-domain approach has improved performance and robustness in comparison to purely frequency domain processing. Computational complexity is lower as well.
\end{abstract}

Keywords: MIMO OFDM, channel estimation, frequency offset, time-varying systems, extended Kalman filtering

\section{Introduction}

Multiple-input multiple-output (MIMO) systems are a key technology in future high data-rate mobile communications. MIMO systems make use of several antennas at the transmitter and receiver sides in order to improve the spectral efficiency and link reliability of the system. Multi-carrier techniques are another important broadband wireless transmission technology. The data is transmitted on several parallel narrowband subcarriers. Combining MIMO with multicarrier techniques provides a feasible physical layer technology for future beyond $3 \mathrm{G}$ and $4 \mathrm{G}$ wireless communications.

One particular multicarrier technique which has received a lot of attention in recent years is orthogonal frequency division multiplexing (OFDM), see e.g. [1]. OFDM transmission turns a frequency selective channel into a set of parallel narrowband channels. This leads to very simple equalization since the transmission becomes free of intersymbol interference (ISI). However, the theoretical benefits of MIMO and OFDM systems may not be fully achieved in broadband mobile applications because the channels are time and frequency selective. In order to enjoy all the advantages of OFDM transmission, two key tasks must be successfully accomplished: channel estimation and frequency synchronization. In high speed mobile scenarios the channel is varying rapidly and the task is not only to estimate the channel accurately but also to track it over time in order to achieve target error rates. One of the main drawbacks of OFDM is its high sensitivity to frequency offsets caused by oscillator inaccuracies, separate radio frequency-intermediate frequency (RF-IF) chains and Doppler shifts due to mobility. These result in inter-carrier interference, leading to severe performance losses especially at high data-rates. The degradation is significant because the number of subcarriers is typically large 
and the inter-carrier spacing is small. Hence, the compensation for the frequency offsets is crucial.

Channel estimation for OFDM systems has been investigated in both single input single output (SISO) systems [2-4] and MIMO case [5-8]. Typically the channels are assumed to be time-invariant. Time-varying channels have been considered in $[6,7,9,10]$. Commonly, the problems of channel and frequency offset estimation are addressed separately [11]. Recursive estimators such as Kalman filter that are suitable for tracking time-varying systems have been used in OFDM, for instance, to estimate the wireless channel in frequency domain [2, 12]. A time-domain approach based on Kalman filter was proposed in [13, 14].

Frequency offset estimation problem was considered in [15]. Extended Kalman filter (EKF) was applied in [16] to jointly estimate code delay and multipath channel parameters in the context of CDMA systems. The idea of joint channel and frequency offset estimation using EKF was proposed for SISO-OFDM systems [17] and extended to MIMO systems in [18].

In this paper, the problem of tracking both time-varying frequency offsets and channel coefficients in MIMO-OFDM systems is addressed. The channel is assumed to be both time and frequency selective. The proposed method performs the channel and carrier frequency offset (CFO) estimation in time-domain followed by equalization in frequency domain. Expressions for both zero-forcing and minimum mean square error (MMSE) equalizers are given. The scope of this paper is channel and CFO tracking. Equalization is by no means optimized, hence the presented bit error rate performance is for illustrative purpose. Each transmitterreceiver pair introduces its own frequency offset [19], induced by mobility together with large angle spread and rich scattering environments, typically assumed in MIMO systems. The estimator is implemented using extended Kalman filtering. A few known training symbols at the beginning of the transmission are used to acquire estimates of the channel and offset parameters, and these parameters are tracked over time. Then, the algorithm may switch to decision-directed (DD) mode and use the decoded symbols for keeping the track, in particular in low mobility scenarios. In high mobility cases, pilot signals are naturally needed [8]. The proposed time-domain approach shows highly reliable tracking performance and robustness. Both the time-varying MIMO channels and the frequency offsets are accurately tracked, with reduced pilot information due to decision-directed processing. Computational complexity is significantly reduced compared to Kalman filter-based tracking in frequency domain.

The rest of the paper is organized as follows. A brief overview of the MIMO-OFDM system model is given next. In Section 3, we derive the nonlinear state-variable model which describes the dynamic behavior of the MIMO channel matrix and frequency offsets. These channel parameters may then be estimated using EKF. Description of the proposed channel and CFO tracking algorithm is given. In Section 4, we show simulation results using time and frequency selective channels and time-varying frequency offsets. Finally, Section 5 concludes the paper.

\section{System Model}

In this section, the T-transmit R-receive MIMO system model employed throughout this paper is described. First, MIMO channel and frequency offset parameters are briefly presented. Then, input-output relationships are given for MIMO-OFDM systems experiencing frequency offset in addition to time and frequency selective fading. Finally, zero forcing and minimum mean square error equalizers operating in frequency domain are presented. 


\subsection{MIMO CHANNEL}

Considering a T-R MIMO system, in the following index $t=1, \ldots, T$ refers to transmit antennas and $r=1, \ldots, R$ to receive antennas. We will write $t r$ when referring to the transmission from antenna $t$ to antenna $r$.

For OFDM with proper cyclic prefix (CP) and timing, the discrete channel frequency response between transmit antenna $t$ and receive antenna $r$ can be expressed as:

$$
H_{t r}[k, n]=\sum_{l=0}^{L-1} h_{t r}[k, l] e^{-j \frac{2 \pi l n}{N}}
$$

where $k$ refers to the discrete time instance, $n=0, \ldots, N-1$ is the subcarrier index and $N$ is the number of subcarriers. There are a total number $L$ of propagation paths. The discrete time channel impulse response between transmit antenna $t$ and receive antenna $r, h_{t r}[k$, $l$ ], is sampled from the continuous time impulse response at time $k T_{f}$ and delay lag $l T_{s} / N$. The terms $T_{f}$ and $T_{s}$ denote the total duration of the OFDM block and of the data part, respectively. In OFDM, the subcarrier spacing is chosen as $\Delta f=1 / T_{s}$. The channel coefficients $h_{t r}[k, l]$ are assumed to be complex zero mean Gaussian random variables, which leads to Rayleigh fading. Each channel tap is considered to be correlated in time. The average power and delay profiles, $\left\{E\left[\left|h_{t r}[k, l]\right|^{2}\right]\right\}_{l=0}^{L-1}$ and $\left\{\tau_{l}\right\}_{i=0}^{L-1}$, respectively, are determined by the environment. Specific commonly occurring scenarios are used in simulations in Section 4.

By assuming block fading model [20], the channel remains constant over one OFDM symbol. The taps of each individual MIMO channel of length $L$ can be stacked into a vector as follows:

$$
\begin{aligned}
\mathbf{h}_{t r}(k) & =\left[h_{t r}[k, 0], h_{t r}[k, 1], \ldots, h_{t r}[k, L-1]\right]^{\mathrm{T}} \\
& =\left[h_{t r}^{0}(k) \ldots h_{t r}^{L-1}(k)\right]^{\mathrm{T}},
\end{aligned}
$$

with $h_{t r}^{l}(k) \triangleq h_{t r}[k, l], l=0, \ldots, L-1$.

Now, we may stack the elements of the whole MIMO channel matrix at time instance $k$ into a column vector of size TRL $\times 1$ :

$$
\mathbf{h}(k)=\left[\mathbf{h}_{11}^{\mathrm{T}}(k) \ldots \mathbf{h}_{T 1}^{\mathrm{T}}(k) \ldots \mathbf{h}_{1 r}^{\mathrm{T}}(k) \ldots \mathbf{h}_{t r}^{\mathrm{T}}(k) \ldots \mathbf{h}_{T r}^{\mathrm{T}}(k) \ldots \mathbf{h}_{1 R}^{\mathrm{T}}(k) \ldots \mathbf{h}_{\mathrm{TR}}^{\mathrm{T}}(k)\right]^{\mathrm{T}} .
$$

Channels corresponding to different transmit and receive antenna pairs in MIMO systems usually exhibit similar delay profiles. Moreover, channel impulse responses are considered to be independent and identically distributed. In practice this means that the scattering environment is rich and the antennas are placed further than the coherence distance apart.

\subsection{Frequency Offsets in Mobile Mimo Systems}

Frequency offsets are mainly caused by two different factors [21]: Doppler shifts and carrier frequency mismatches between transmit and receive oscillators.

Carrier frequency mismatches occur when oscillators at the transmitter and receiver side experience drifts from their nominal frequency. Therefore, a frequency offset is introduced. In 
multi-antenna systems each transmitter and receiver typically requires its own radio frequencyintermediate frequency (RF-IF) chain. Consequently, each transmitter-receiver pair has its own mismatch parameter and hence separate frequency offset. In a $T \times R$ MIMO system this leads to $T \times R$ mismatch parameters and offsets. If transmit or receive antennas share RF-IF chains, fewer carrier mismatch parameters are needed.

In wireless mobile communications, Doppler shift arises from relative motion between the transmitter and the receiver. This leads to a shift of the received signal spectrum. The shift depends on the carrier frequency and the velocity of the mobile terminal [22-25]. In theory, separate offset parameter is needed for each propagation path [22-24]. In MIMO wireless communication systems, rich scattering environment and large angle spread are required in order to obtain improved spectral efficiency and link quality. In case of mobile MIMO systems, each channel branch is assumed to introduce its own Doppler shift. Similar approach is adopted in [19]. The assumption of a single offset per multi-antenna receiver is valid only in the case when multipath components impinge the antennas with the same angle of arrival (AOA) [19, $21,26]$, for example, in the case of Rician channel with very few dominant AOA's. Hence, in a general case of $T \times R$ MIMO system it is necessary to compensate for $T \times \mathrm{R}$ different Doppler shifts.

Just to conclude, using separate frequency offset parameters for each MIMO branch is justified by mobility, rich scattering environment and separate RF-IF chains. In particular scenarios where there is a strong line-of-sight component and shared RF-IF chains, fewer offset parameters may be used. This leads to reduced computational complexity.

\subsection{MiMO OFDM System Model}

In the following, the transmission process is described in a formal way, using the notation introduced in [20]. The MIMO-OFDM system model is depicted in Figure 1.

The $k$-th modulated OFDM block after CP insertion at transmit antenna $t$ is written as $\tilde{\mathbf{x}}_{t}(k)=\mathbf{T}_{\mathrm{CP}} \mathbf{F}_{N} \mathbf{a}_{t}(k)$, where $\mathbf{F}_{N}$ is the $N \times N$ inverse discrete Fourier transform (IDFT) matrix, $N$ is the total number of subcarriers, $\mathbf{a}_{t}(k)$ is the $N \times 1$ complex symbol vector sent from antenna $t$, and $\mathbf{T}_{\mathrm{CP}}$ is the cyclic prefix insertion matrix defined as:

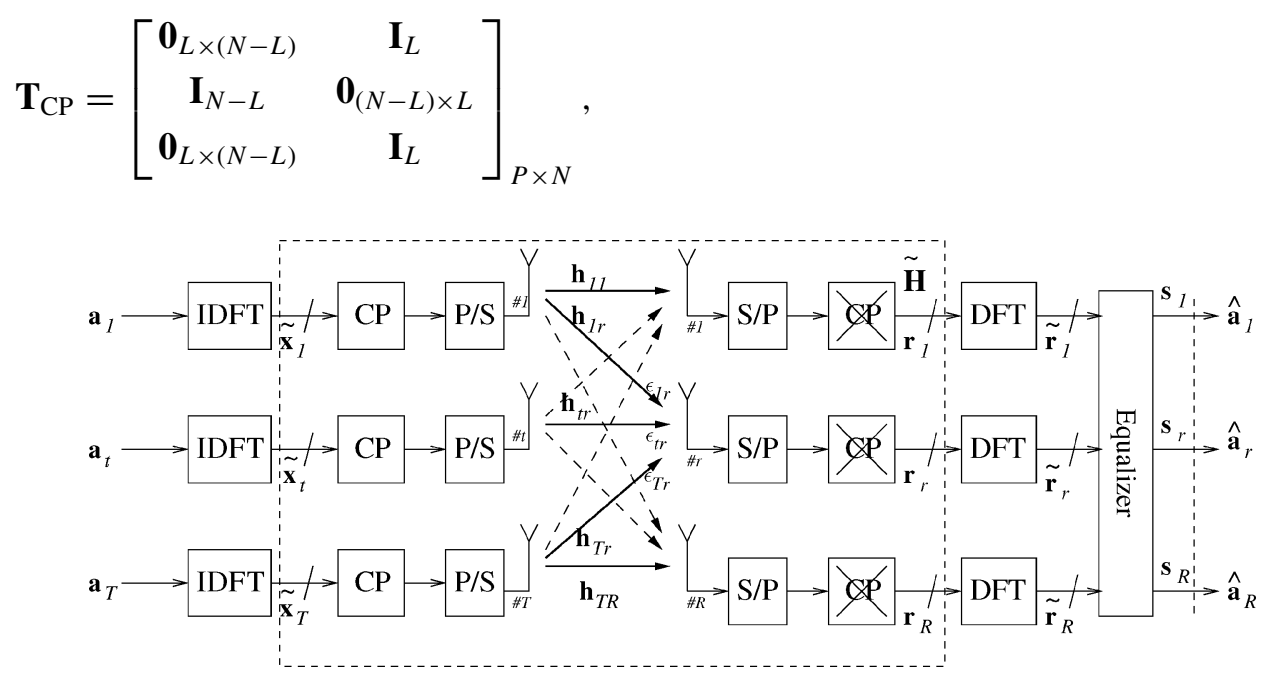

Figure 1. MIMO-OFDM transmission model. 
where $L$ is the length of the cyclic prefix and consequently $P=N+L$ is the total OFDM block length. The notation $\mathbf{I}_{K}$ refers to the identity matrix of size $K \times K$, and $\mathbf{0}_{K \times L}$ to matrix of size $K \times L$ filled with zeros.

Frequency offsets are modeled at the receiver side. This reflects the physical reality, as this modeling accounts for both local oscillator drifts and Doppler shifts induced by mobility. Carrier frequency deviation occurs before the removal of the cyclic prefix, yielding a noncontinuous behavior at symbol borders. As argued in Section 2.2, each MIMO branch is considered to introduce its own frequency offset. In a $T \times R$ MIMO system there are $T \times R$ different offsets to be compensated for.

Introducing the offset at the receiver, before cyclic prefix removal, leads to the following expressions for the received signal in time-domain at the $r$-th antenna after cyclic prefix removal:

$$
\mathbf{r}_{r}(k)=\sum_{t=1}^{T} \mathbf{R}_{\mathrm{CP}} \mathbf{C}_{t r}^{\epsilon}(k) \mathbf{H}_{t r}(k) \tilde{\mathbf{x}}_{t}(k)+\mathbf{w}_{r}(k), \quad r=1, \ldots, R,
$$

where $\mathbf{R}_{\mathrm{CP}}=\left[\mathbf{0}_{N \times L} \mathbf{I}_{N}\right]_{N \times P}$ of size $N \times P$ performs cyclic prefix removal on a single OFDM block. $\mathbf{C}_{t r}^{\epsilon}(k)$ is the $P \times P$ frequency offset matrix having the form $\mathbf{C}_{t r}^{\epsilon}(k)=$ $\operatorname{diag}\left\{\exp \left(j \frac{2 \pi n \epsilon_{t r}(k)}{N}\right)\right\}$, with $n=0, \ldots, P-1$. The quantities $\epsilon_{t r}(k)$, chosen as $0 \leq \epsilon_{t r}(k)<$ 1 , are referred to as normalized frequency offsets, that is effective frequency deviation lies in the interval $[0, B / N[$, where $B$ is the total bandwidth allocated to the system. The matrix $\mathbf{H}_{t r}(k)$ models the wireless environment between the $t$-th transmit and $r$-th receive antenna at OFDM block time $k$. Each $\mathbf{H}_{t r}(k)$ is a Toeplitz convolution matrix of size $P \times P$ made out of the channel vector $\mathbf{h}_{t r}(k)$. The maximum channel length is $L$ and the length of the cyclic prefix is also set equal to $L$ in order to avoid inter-block interference. The TR channel vectors $\mathbf{h}_{t r}(k)$ are written as described at the end of Section 2.1, i.e. $\mathbf{h}_{t r}(k)=\left[h_{t r}^{0}(k) \ldots h_{t r}^{L-1}(k)\right]^{\mathrm{T}}$. The channel taps $\left\{h_{t r}^{l}(k)\right\}_{l=0, \ldots, L-1}$ are assumed to be invariant over the duration of one ODFM block and they are assumed to vary independently in time. The additive white Gaussian noise vector $\mathbf{w}_{r}(k)$ has covariance matrix $\sigma^{2} \mathbf{I}_{N}$.

Equivalently, Equation (6) can be written in the following form:

$$
\mathbf{r}_{r}(k)=\sum_{t=1}^{T} \mathbf{R}_{\mathrm{CP}} \mathbf{C}_{t r}^{\epsilon}(k) \tilde{\mathbf{X}}_{t}(k) \mathbf{h}_{t r}(k)+\mathbf{w}_{r}(k), \quad r=1, \ldots, R,
$$

where $\tilde{\mathbf{X}}_{t}(k)$ are Toeplitz matrices of dimension $P \times L$ built from the vector $\tilde{\mathbf{x}}_{t}(k), \quad t=$ $1, \ldots, T$.

The set of $R$ equations in (6) or (7) may be written in more compact matrix form. The received $R N \times 1$ signal block after cyclic prefix insertion, followed by transmission on the wireless channel and cyclic prefix removal may then be written as follows:

$$
\begin{aligned}
\mathbf{r}(k) & =\tilde{\mathbf{R}}_{\mathrm{CP}} \mathbf{H}^{\epsilon}(k) \tilde{\mathbf{x}}(k)+\mathbf{w}(k) \\
& =\tilde{\mathbf{R}}_{\mathrm{CP}} \tilde{\mathbf{X}}(k) \mathbf{h}(k)+\mathbf{w}(k),
\end{aligned}
$$

where $\mathbf{r}(k)=\left[\mathbf{r}_{1}^{T}(k) \ldots \mathbf{r}_{r}^{T}(k) \ldots \mathbf{r}_{R}^{T}(k)\right]^{T}$ is the $k$-th received block of size $R N \times 1$ at the $R$ receive antennas, $\tilde{\mathbf{x}}(k)=\left[\tilde{\mathbf{x}}_{1}^{T}(k) \ldots \tilde{\mathbf{x}}_{t}^{T}(k) \ldots \tilde{\mathbf{x}}_{T}^{T}(k)\right]^{T}$ is the $k$-th OFDM modulated block of size $T P \times 1$ at the $T$ transmit antennas, and $\mathbf{w}(k)=\left[\mathbf{w}_{1}^{T}(k) \ldots \mathbf{w}_{r}^{T}(k) \ldots \mathbf{w}_{R}^{T}(k)\right]^{T}$ is the circular complex white Gaussian noise vector with covariance matrix $\mathbf{R}_{\mathbf{w}}=\sigma^{2} \mathbf{I}_{R N}$. The 
channel vectors corresponding to each MIMO branch are stacked into the vector

$$
\mathbf{h}(k)=\left[\mathbf{h}_{11}^{T}(k) \ldots \mathbf{h}_{T 1}^{T}(k) \ldots \mathbf{h}_{1 r}^{T}(k) \ldots \mathbf{h}_{t r}^{T}(k) \ldots \mathbf{h}_{T r}^{T}(k) \ldots \mathbf{h}_{1 R}^{T}(k) \ldots \mathbf{h}_{\mathrm{TR}}^{T}(k)\right]^{T} .
$$

The block diagonal matrix $\tilde{\mathbf{R}}_{\mathrm{CP}}$ of size $R N \times R P$ is designed to remove the cyclic prefix from the $R P \times 1$ signal block at the receive antennas and is defined as:

$$
\tilde{\mathbf{R}}_{\mathrm{CP}}=\left[\begin{array}{cccc}
\mathbf{R}_{\mathrm{CP}} & \mathbf{0}_{N \times P} & \cdots & \mathbf{0}_{N \times P} \\
\mathbf{0}_{N \times P} & \mathbf{R}_{\mathrm{CP}} & & \vdots \\
\vdots & & \ddots & \mathbf{0}_{N \times P} \\
\mathbf{0}_{N \times P} & \cdots & \mathbf{0}_{N \times P} & \mathbf{R}_{\mathrm{CP}}
\end{array}\right]_{R N \times R P} .
$$

The $R P \times T P$ MIMO channel convolution matrix in (8), which takes into account also the frequency offset on each MIMO branch, may be expressed as:

$$
\mathbf{H}^{\epsilon}(k)=\left[\begin{array}{ccc}
\mathbf{H}_{11}^{\epsilon}(k) & \cdots & \mathbf{H}_{T 1}^{\epsilon}(k) \\
\vdots & \mathbf{H}_{t r}^{\epsilon}(k) & \vdots \\
\mathbf{H}_{1 R}^{\epsilon}(k) & \cdots & \mathbf{H}_{\mathrm{TR}}^{\epsilon}(k)
\end{array}\right]_{R P \times T P},
$$

where we defined the matrices $\mathbf{H}_{t r}^{\epsilon}(k) \triangleq \mathbf{C}_{t r}^{\epsilon}(k) \mathbf{H}_{t r}(k), t=1, \ldots, T, r=1, \ldots, R$.

If model (9) is employed, the data and offset matrix of size $R P \times T R L$ is the following:

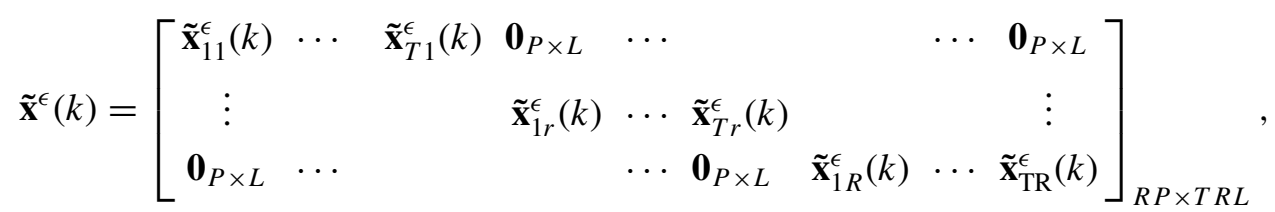

where sub-matrices $\tilde{\mathbf{X}}_{t r}^{\epsilon}(k)$ are defined as $\tilde{\mathbf{X}}_{t r}^{\epsilon}(k) \triangleq \mathbf{C}_{t r}^{\epsilon}(k) \tilde{\mathbf{X}}_{t}(k), t=1, \ldots, T, r=1, \ldots, R$.

OFDM demodulation is performed via discrete Fourier transform (DFT) at each receive antenna. Taking the DFT of $\mathbf{r}_{r}(k)$ as defined in (6) yields:

$$
\tilde{\mathbf{r}}_{r}(k)=\sum_{t=1}^{T} \mathbf{F}_{N}^{H} \mathbf{R}_{\mathrm{CP}} \mathbf{C}_{t r}^{\epsilon}(k) \mathbf{H}_{t r}(k) \tilde{\mathbf{x}}_{t}(k)+\mathbf{F}_{N}^{H} \mathbf{w}_{r}(k),
$$

where $\mathbf{F}_{N}^{H}$ is unitary DFT matrix. Equation (13) can be further written as:

$$
\tilde{\mathbf{r}}_{r}(k)=\sum_{t=1}^{T} \mathbf{F}_{N}^{H} \mathbf{R}_{\mathrm{CP}} \mathbf{C}_{t r}^{\epsilon}(k) \mathbf{H}_{t r}(k) \mathbf{T}_{\mathrm{CP}} \mathbf{F}_{N} \mathbf{a}_{t}(k)+\mathbf{F}_{N}^{H} \mathbf{w}_{r}(k) .
$$

Let us define for any $t, r$ the following matrix of size $N \times N$ :

$$
\mathbf{M}_{t r}(k)=\mathbf{F}_{N}^{H} \mathbf{R}_{\mathrm{CP}} \mathbf{C}_{t r}^{\epsilon}(k) \mathbf{H}_{t r}(k) \mathbf{T}_{\mathrm{CP}} \mathbf{F}_{N} .
$$

With the notation introduced in (15), equation (14) becomes:

$$
\tilde{\mathbf{r}}_{r}(k)=\sum_{t=1}^{T} \mathbf{M}_{t r}(k) \mathbf{a}_{t}(k)+\tilde{\mathbf{n}}_{r}(k),
$$


where $\tilde{\mathbf{n}}_{r}(k)=\mathbf{F}_{N}^{H} \mathbf{w}_{r}(k)$.

Now, we have the two cases depending on the frequency offset. If $\epsilon_{t r}(k)=0$ : then $\mathbf{C}_{t r}^{\epsilon}(k) \equiv$ $\mathbf{I}_{p}$ and the matrix $\mathbf{R}_{\mathrm{CP}} \mathbf{C}_{t r}^{\epsilon}(k) \mathbf{H}_{t r}(k) \mathbf{T}_{\mathrm{CP}}=\mathbf{R}_{\mathrm{CP}} \mathbf{H}_{t r}(k) \mathbf{T}_{\mathrm{CP}} \triangleq \tilde{\mathbf{H}}_{t r}(k)$ becomes circulant. Since circulant matrices implement circular convolutions, they are diagonalized by DFT and IDFT operations. Then, the following holds:

$$
\begin{aligned}
\mathbf{M}_{t r}(k) & =\mathbf{F}_{N}^{H} \tilde{\mathbf{H}}_{t r}(k) \mathbf{F}_{N} \\
& =\operatorname{diag}\left\{\sum_{l=0}^{L-1} h_{t r}^{l}(k) \exp \left(-j \frac{2 \pi n l}{N}\right)\right\}_{n=0, \ldots, N-1} .
\end{aligned}
$$

Thus $\mathbf{M}_{t r}(k)$ is diagonal and contains the frequency response of the channel $\mathbf{h}_{t r}(k)$ at each subcarrier frequency. The transmission becomes free of intercarrier interference (ICI). Only the frequency flat fading on each subcarrier needs to be compensated for, in order to decode the transmitted symbols.

In the case carrier frequency offset takes place (may be due to mismatch or rich scattering in addition to mobility), $0<\epsilon_{t r}(k)<1$ and the matrix $\mathbf{R}_{\mathrm{CP}} \mathbf{C}_{t r}^{\epsilon}(k) \mathbf{H}_{t r}(k) \mathbf{T}_{\mathrm{CP}}$ is not circulant anymore. Hence $\mathbf{M}_{t r}(k)$ is not a diagonal matrix anymore, which leads to ICI and the orthogonality property of the OFDM transmission is lost. In this case both the channel and the frequency offset need to be compensated for.

Now, stacking the $R$ equations in (16) in a vector we obtain:

$$
\tilde{\mathbf{r}}(k) \triangleq\left[\tilde{\mathbf{r}}_{1}^{T}(k) \ldots \tilde{\mathbf{r}}_{R}^{T}(k)\right]^{T}=\mathbf{M}(k) \mathbf{a}(k)+\tilde{\mathbf{n}}(k),
$$

where $\mathbf{a}(k)=\left[\mathbf{a}_{1}^{T}(k) \ldots \mathbf{a}_{t}^{T}(k) \ldots \mathbf{a}_{T}^{T}(k)\right]^{T}, \tilde{\mathbf{n}}(k)=\left[\tilde{\mathbf{n}}_{1}^{T}(k) \ldots \tilde{\mathbf{n}}_{r}^{T}(k) \ldots \tilde{\mathbf{n}}_{R}^{T}(k)\right]^{T}$ and the $R N \times$ $T N$ matrix $\mathbf{M}(k)$ is:

$$
\mathbf{M}(k)=\left[\begin{array}{ccccc}
\mathbf{M}_{11}(k) & \cdots & \ldots & \cdots & \mathbf{M}_{T 1}(k) \\
\vdots & & \mathbf{M}_{t r}(k) & & \vdots \\
\mathbf{M}_{1 R}(k) & \cdots & \cdots & \cdots & \mathbf{M}_{\mathrm{TR}}(k)
\end{array}\right]_{R N \times T N} .
$$

The equalization may be performed in frequency domain as follows:

$$
\begin{aligned}
\mathbf{u}(k) & =\mathbf{U}(k) \tilde{\mathbf{r}}(k) \\
& =\mathbf{U}(k)[\mathbf{M}(k) \mathbf{a}(k)+\tilde{\mathbf{n}}(k)],
\end{aligned}
$$

where $\mathbf{U}(k)$ is the $T N \times N$ equalizer matrix at OFDM block time $k$. Then, decisions are carried out on $\mathbf{u}(k)$ in order to obtain the symbol estimate $\hat{\mathbf{a}}(k)$. The zero forcing and MMSE equalizers may be found as follows:

1. The zero forcing (ZF) equalizer is given by:

$$
\mathbf{U}_{\mathrm{ZF}}(k)=\mathbf{M}^{+}(k),
$$

where $\mathbf{M}^{+}=\left(\mathbf{M}^{H} \mathbf{M}\right)^{-1} \mathbf{M}^{H}$ is the left pseudoinverse of $\mathbf{M}$.

2. MMSE equalizer may be found as follows:

$$
\mathbf{U}_{\mathrm{MMSE}}(k)=\sigma_{a}^{2} \mathbf{M}^{H}(k)\left[\sigma_{a}^{2} \mathbf{M}(k) \mathbf{M}^{H}(k)+\sigma^{2} \mathbf{I}\right]^{-1},
$$

where $\sigma^{2}=\sigma_{\tilde{n}}^{2}$ is the variance of the noise and $\sigma_{a}^{2}$ is the average symbol energy. 
In order to ensure data symbol detectability with zero forcing, $\mathbf{M}^{H} \mathbf{M}$ has to be full rank, i.e. $T N=\operatorname{rank}\left\{\mathbf{M}^{H} \mathbf{M}\right\}=\operatorname{rank}\{\mathbf{M}\} \leq \min (T N, R N)$. Hence $\min (T N, R N)=T N$, i.e. the number of receive antennas must be at least equal with the number of transmit antennas.

MMSE and zero-forcing schemes were presented just to give the reader some indication about the bit error rate performance. The equalization stage is by no means optimized in this paper, since the scope is channel and $\mathrm{CFO}$ tracking. However, there exist number of equalization schemes in the literature [27, 28], which can be fitted to the state-space framework we have developed. Those may provide with better end-user performance, meaning improved bit error rate and potentially reduced complexity.

\section{Channel and Offset Estimation and Tracking}

In mobile MIMO wireless communications, the channels are time-varying, and mobility combined with rich scattering induces multiple carrier frequency offsets. Hence, channel and offset parameters need to be tracked and equalizer coefficients updated periodically. In this section, we derive a method for estimating and tracking both the MIMO channel coefficients and the frequency offsets over time. The method stems from extended Kalman filtering (EKF). The frequency offsets have been both modeled and simulated at the receiver side throughout this paper.

We start by writing the state-variable model. The linear state equation is the following:

$$
\mathbf{s}(k)=\mathbf{A s}(k-1)+\mathbf{v}(k),
$$

where the state vector $\mathbf{s}(k)=\left[\mathbf{h}(k)^{T} \boldsymbol{\epsilon}(k)^{T}\right]^{T}$ is comprised of channel coefficients $\mathbf{h}(k)$ and frequency offsets values:

$$
\boldsymbol{\epsilon}(k)=\left[\epsilon_{11}(k) \ldots \epsilon_{T 1}(k) \ldots \epsilon_{1 r}(k) \ldots \epsilon_{T r}(k) \ldots \epsilon_{1 R}(k) \ldots \epsilon_{\mathrm{TR}}(k)\right]^{\mathrm{T}} .
$$

There are $T R L$ channel taps and $T R$ frequency offset values in the state vector of dimension $T R(L+1)$. The state transition matrix $\mathbf{A}$ is of size $T R(L+1) \times T R(L+1)$. In this paper $\mathbf{A}$ is considered to be close to the identity matrix $\left(\mathbf{A}=a \mathbf{I}_{\mathrm{TR}(L+1)}, a=0.99\right)$. The state transition matrix describes the dynamics of the state vector, i.e. its time auto-correlation properties. Low order auto-regressive (AR) models are widely and practically used in the literature [16, 29]. Elements of $\mathbf{A}$ are shown to be related to the Doppler frequency [16]. The part of the state vector containing the channel coefficients may also be augmented to model an AR process of order $p[29,30]$. The process covariance matrix $\mathbf{Q}_{s}$ has the following structure:

$$
\mathbf{Q}_{\mathbf{s}}=\left[\begin{array}{cc}
\mathbf{Q}_{h} & \mathbf{0}_{T R L \times T R} \\
\mathbf{0}_{T R L \times T R} & \mathbf{Q}_{\epsilon}
\end{array}\right]_{T R(L+1) \times T R(L+1)},
$$

where $\mathbf{Q}_{h}=\sigma_{h}^{2} \mathbf{I}_{\mathrm{TRL}}$ and $\sigma_{h}^{2}$ is the variance of the state noise associated with channel coefficients. $\mathbf{Q}_{\epsilon}=\sigma_{\epsilon}^{2} \mathbf{I}_{\mathrm{TR}}$ and $\sigma_{\epsilon}^{2}$ is the variance of the state noise associated with offsets.

We recall the measurement equation (8) or (9) where the $R N \times R N$ covariance matrix of the measurement noise $\mathbf{w}$ is given by $\mathbf{R w}=\sigma^{2} \mathbf{I}_{R N}$. The noise statistics $\left(\mathbf{R}_{\mathrm{w}}\right.$ and $\left.\mathbf{Q}_{s}\right)$ are considered to be known. The parameters $\mathbf{A}, \mathbf{Q}_{s}$ and $\mathbf{R}_{w}$ can, however, be reliably estimated from the received data, see e.g. [31].

Equations (8) and (9) are linear in the channel coefficients, and non-linear in the offset values. Both these forms of the measurement equation will be needed later in the derivations. Due 
to nonlinearities introduced by frequency offsets, the measurement equation can be rewritten as:

$$
\mathbf{r}(k)=\mathbf{g}(\mathbf{s}(k))+\mathbf{w}(k),
$$

where g: $\mathbb{C}_{T R(L+1)} \rightarrow \mathbb{C}_{R N}$ defined as $\mathbf{g}:$

$$
\mathbf{s}(k) \triangleq\left[\begin{array}{c}
\mathbf{h}(k) \\
\epsilon(k)
\end{array}\right] \mapsto \mathbf{g}(\mathbf{s}(k))=\tilde{\mathbf{R}}_{\mathrm{CP}} \mathbf{H}^{\epsilon}(k) \tilde{\mathbf{x}}(k)=\tilde{\mathbf{R}}_{\mathrm{CP}} \tilde{\mathbf{X}}^{\epsilon}(k) \mathbf{h}(k)
$$

is a non-linear function of the state vector $\mathbf{s}$.

For simplicity of the notation, the time index $k$ will be omitted in the set equations (29)-(42). Given the linear and non-linear state and measurement equations, we have to define the Jacobian $\partial \mathbf{g} / \partial \mathbf{s}$ in order to apply EKF. Taking into account the structure of the state vector, the Jacobian matrix $\mathbf{G}$ of $\mathbf{g}$ is the following:

$$
\mathbf{G} \triangleq \frac{\partial \mathbf{g}}{\partial \mathbf{s}}=\left[\frac{\partial \mathbf{g}}{\partial \mathbf{h}}, \frac{\partial \mathbf{g}}{\partial \boldsymbol{\epsilon}}\right]_{R N \times T R(L+1)} .
$$

Hence $\partial \mathbf{g} / \partial \mathbf{s}$ can be split into two parts, the derivative with respect to $\mathbf{h}$ and the one with respect to $\epsilon$. We will now derive expressions for these two quantities. Using the second formulation for $\mathbf{g}$ in (28) we obtain:

$$
\begin{aligned}
\frac{\partial \mathbf{g}}{\partial \mathbf{h}} & \triangleq\left[\frac{\partial \mathbf{g}}{\partial \mathbf{h}_{11}} \cdots \frac{\partial \mathbf{g}}{\partial \mathbf{h}_{T 1}} \cdots \frac{\partial \mathbf{g}}{\partial \mathbf{h}_{1 r}} \cdots \frac{\partial \mathbf{g}}{\partial \mathbf{h}_{T r}} \cdots \frac{\partial \mathbf{g}}{\partial \mathbf{h}_{1 R}} \cdots \frac{\partial \mathbf{g}}{\partial \mathbf{h}_{\mathrm{TR}}}\right]_{R N \times T R L} \\
& =\left[\frac{\partial}{\partial \mathbf{h}}\left(\tilde{\mathbf{R}}_{\mathrm{CP}} \tilde{\mathbf{X}}^{\epsilon} \mathbf{h}\right)\right]_{R N \times T R L} \\
& =\tilde{\mathbf{R}}_{\mathrm{CP}} \tilde{\mathbf{X}}^{\epsilon}
\end{aligned}
$$

The derivative of $\mathbf{g}$ with respect to vector $\epsilon$ is defined as:

$$
\frac{\partial \mathbf{g}}{\partial \epsilon} \triangleq\left[\frac{\partial \mathbf{g}}{\partial \epsilon_{11}} \cdots \frac{\partial \mathbf{g}}{\partial \epsilon_{T 1}} \cdots \frac{\partial \mathbf{g}}{\partial \epsilon_{1 r}} \cdots \frac{\partial \mathbf{g}}{\partial \epsilon_{T r}} \cdots \frac{\partial \mathbf{g}}{\partial \epsilon_{1 R}} \cdots \frac{\partial \mathbf{g}}{\partial \epsilon_{\mathrm{TR}}}\right]_{R N \times T R} .
$$

Recalling from (6) the expression for the received signal in time-domain at the $r$-th antenna, the $r$-th vector block of $\mathbf{g}=\left[\mathbf{g}_{1}^{T}, \ldots, \mathbf{g}_{r}^{T}, \ldots, \mathbf{g}_{R}^{T}\right]^{\mathrm{T}}$ is written as:

$$
\mathbf{g}_{r}=\sum_{t=1}^{T} \mathbf{R}_{\mathrm{CP}} \mathbf{C}_{t r}^{\epsilon} \mathbf{H}_{t r} \tilde{\mathbf{x}}_{t}, \quad r=1, \ldots, R .
$$

First, by differentiating (34) with respect to $\epsilon_{k l}$ one obtains:

$$
\frac{\partial \mathbf{g}_{r}}{\partial \epsilon_{k l}}=\left\{\begin{array}{ll}
\mathbf{R}_{\mathrm{CP}} \tilde{\mathbf{C}}_{k r}^{\epsilon} \mathbf{y}_{k r}, & \text { if } l=r \\
\mathbf{0}_{P \times 1}, & \text { otherwise. }
\end{array} \quad k=1, \ldots, T, \quad l=1, \ldots, R,\right.
$$

with the following definitions:

$$
\mathbf{y}_{t r} \triangleq \mathbf{H}_{t r} \tilde{\mathbf{x}}_{t}
$$


and

$$
\begin{aligned}
\tilde{\mathbf{C}}_{t r}^{\epsilon} & \Delta \operatorname{diag}\left\{\frac{\partial e^{\frac{j 2 \pi n \epsilon_{t r}}{N}}}{\partial \epsilon_{t r}}\right\}_{n=0, \ldots, P-1} \\
& =\left[\begin{array}{cccc}
0 & 0 & \ldots & 0 \\
0 & \frac{j 2 \pi}{N} e^{\frac{j 2 \pi \epsilon_{t r}}{N}} & \vdots \\
\vdots & & \ddots & 0 \\
0 & \ldots & 0 & \frac{j 2 \pi(P-1)}{N} e^{\frac{j 2 \pi \epsilon_{t r}(P-1)}{N}}
\end{array}\right]_{P \times P} .
\end{aligned}
$$

Then, by stacking the $R$ vectors in (35) into a column vector,

$$
\frac{\partial \mathbf{g}}{\partial \epsilon_{k l}}=\left[\begin{array}{c}
\frac{\partial \mathbf{g}_{1}}{\partial \epsilon_{k l}} \\
\vdots \\
\frac{\partial \mathbf{g}_{R}}{\partial \epsilon_{k l}}
\end{array}\right]
$$

Finally, the differential $\partial \mathbf{g} / \partial \epsilon$ in (33) may be expressed in a compact form as:

$$
\frac{\partial \mathbf{g}}{\partial \epsilon}=\tilde{\mathbf{R}}_{\mathrm{CP}} \overline{\mathbf{Y}}^{\epsilon},
$$

with

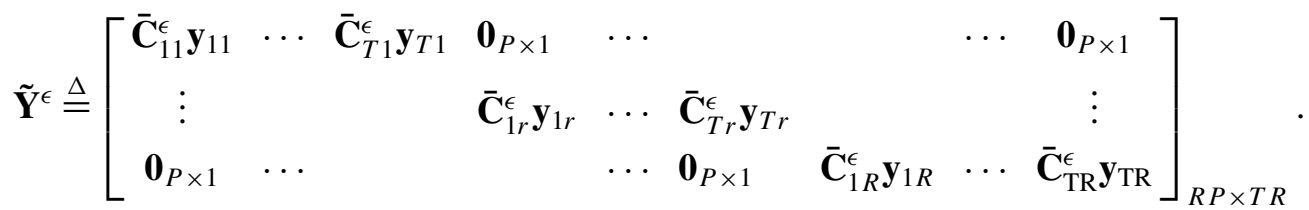

Having now the two differentials $\partial \mathbf{g} / \partial \mathbf{h}$ and $\partial \mathbf{g} / \partial \epsilon$, it remains to construct the matrix $\mathbf{G}$ as in formula (29), which yields the following Jacobian matrix:

$$
\mathbf{G}=\left[\tilde{\mathbf{R}}_{\mathrm{CP}} \tilde{\mathbf{X}}^{\epsilon}, \tilde{\mathbf{R}}_{\mathrm{CP}} \tilde{\mathbf{Y}}^{\epsilon}\right]_{R N \times T R(L+1)} .
$$

Using the expression of $\mathbf{G}$ given in (42), EKF can be applied using the well-known Kalman equations (43)-(47):

$$
\begin{aligned}
\hat{\mathbf{s}}_{(k \mid k-1)} & =\mathbf{A} \hat{\mathbf{s}}_{(k-1 \mid k-1)}, \\
\mathbf{P}_{(k \mid k-1)} & =\mathbf{A} \mathbf{P}_{(k-1 \mid k-1)} \mathbf{A}^{T}+\mathbf{Q}_{\mathbf{s}}, \\
\mathbf{K}_{(k)} & =\mathbf{P}_{(k \mid k-1)} \mathbf{G}_{(k)}^{H}\left[\mathbf{G}_{(k)} \mathbf{P}_{(k \mid k-1)} \mathbf{G}_{(k)}^{H}+\mathbf{R}_{\mathbf{w}}\right]^{-1}, \\
\mathbf{P}_{(k \mid k)} & =\left[\mathbf{I}-\mathbf{K}_{(k)} \mathbf{G}_{(k)}\right] \mathbf{P}_{(k \mid k-1)}, \\
\hat{\mathbf{s}}_{(k \mid k)} & =\hat{\mathbf{s}}_{(k \mid k-1)}+\mathbf{K}_{(k)}\left[\mathbf{r}(k)-\mathbf{g}\left(\hat{\mathbf{s}}_{(k \mid k-1)}\right)\right] .
\end{aligned}
$$

As previously mentioned, channel and offset estimation are done in time-domain while the equalization is performed in frequency domain. The overall structure of the receiver is illustrated in Figure 2. The algorithm works as follows. For each OFDM symbol at time $k$ : 


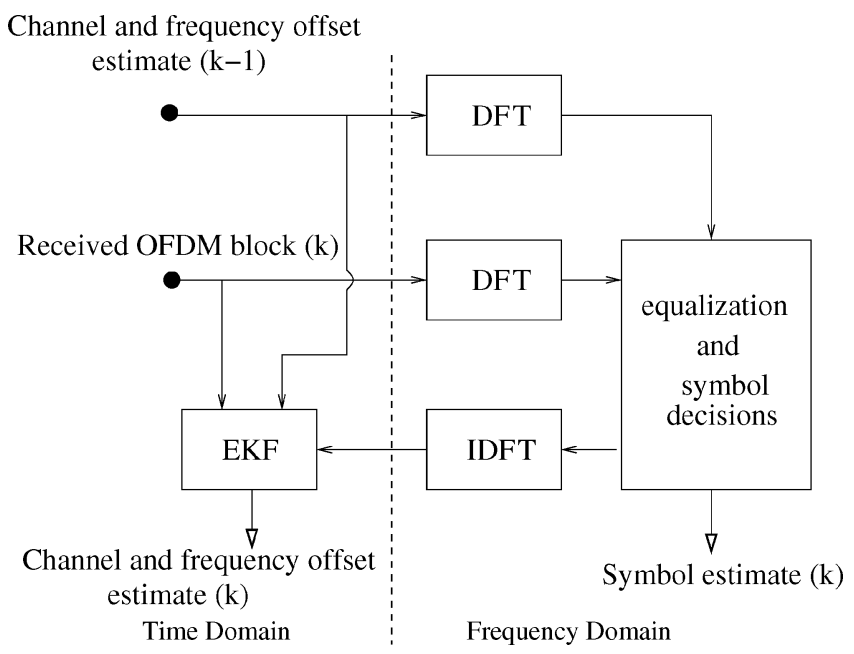

Figure 2. Time-domain channel and frequency offset estimation and tracking followed by frequency domain equalization.

1. Decode the received vector $\mathbf{r}(k)$ and find the symbol estimate $\hat{\mathbf{a}}(k)$, using $\hat{\mathbf{h}}(k-1 \mid k-1)$ and $\hat{\epsilon}(k-1 \mid k-1)$, i.e. respectively the filtered estimate of the channel and the offset values at symbol time $k-1$.

2. Re-modulate $\hat{\mathbf{a}}_{t}(k): \tilde{\overline{\mathbf{x}}}_{t}(k)=\mathbf{T}_{\mathrm{CP}} \mathbf{F}_{N} \hat{\mathbf{a}}_{t}(k), t=1, \ldots, T$.

3. Build the estimates $\hat{\tilde{\mathbf{X}}}^{\epsilon}(k), \hat{\mathbf{H}}^{\epsilon}(k)$ and $\hat{\mathbf{G}}(k)$ using

$$
\hat{\tilde{\mathbf{x}}}(k)=\left[\hat{\tilde{\mathbf{x}}}_{1}^{T}(k) \cdots \hat{\tilde{\mathbf{x}}}_{T}^{T}(k)\right]^{\mathrm{T}},
$$

and predicted estimates $\hat{\mathbf{h}}(k \mid k-1)$ and $\hat{\epsilon}(k \mid k-1)$.

4. Run the extended Kalman filter to finally obtain $\hat{\mathbf{h}}(k \mid k)$ and the estimates of the offsets $\hat{\epsilon}(k \mid k)$.

At this point one may also refine the estimates by re-decoding the symbol $\mathbf{a}(k)$ using the filtered estimate $\hat{\mathbf{h}}(k \mid k)$. Substantial gains in performance are achieved as will be shown later in the simulations section. Except for the first few OFDM blocks, the algorithm works in a decision-directed mode. The initialization phase requires a few symbols (typically about half of the state dimension). For example if we have a two-transmit, two-receive MIMO system and the channel memory is of length 4 , the dimension of the state will be 20 . In this case 10 OFDM symbols are enough for EKF to converge in practice.

The major computational cost lies in the calculation of the matrix inversion in the Kalman gain expression (45). By applying the matrix inversion lemma, the number of required operations can be made proportional to $(L+1) N^{2}$ when tracking is done in time-domain. The complexity is proportional to $N^{3}$ when the processing takes place in the frequency domain. In practice $L \ll N$, hence significantly lower complexity is achieved. Smaller parameter space compared to frequency domain tracking $(T R(L+1) \times 1$ versus $T R(N+1) \times 1$ state vector) leads also to smaller variance for the estimated channel values.

Pre-DFT compensation for frequency offsets prior to equalization cannot be performed in our MIMO scenario: having one offset per MIMO branch makes the problem non-separable and equalization for both channels and offsets needs to be done simultaneously. Hence 
due to multiple CFO's, equalization becomes computationally expensive. Pre-DFT compensation is however possible, in case there is only one CFO per multi-antenna receiver. Complexity of the subsequent channel equalizer is then significantly reduced, due to the absence of ICI. The proposed state-space model may be easily modified to any of these scenarios.

\section{Simulations}

In this section, simulation results are reported. We consider the following performance criteria: the normalized mean squared error (NMSE) of the proposed channel and offset estimation method and the raw bit error rate (BER) of the system, since no diversity or coding technique has been applied. Considering the scope of this paper, i.e. channel and CFO estimation, the NMSE yields a relevant performance measure. However, for completeness we also show the raw BER. Illustrative plots demonstrating the high fidelity tracking capability of the proposed algorithm are presented. In our simulation studies, results are presented in the case where frequency offsets are introduced at the receiver side. However, modeling the offset at the transmitter exhibits similar tracking and BER performance.

A two-transmit, two-receive antenna MIMO system is considered. The carrier frequency is $f_{0}=2.4 \mathrm{GHz}$ and the number of subcarriers is set to $N=128$. The available bandwidth is chosen equal to $B=1 \mathrm{MHz}$. The subcarrier symbol rate is of $7.8 \mathrm{KHz}$. QPSK symbol modulation is employed.

The signal to noise ratio (SNR) is defined as:

$$
\mathrm{SNR}=\frac{E\|\tilde{\mathbf{x}}\|^{2}}{E\|\mathbf{w}\|^{2}}=\frac{E_{s}}{N_{0}},
$$

where $E_{s}$ is the symbol energy and $N_{0}$ is the noise variance.

Wireless channels from each transmit to each receive antenna experience Rayleigh fading with independent propagation paths. Various mobile speeds are used throughout the experiments. Precise SNR and speed value are mentioned in the caption of each figure. A receiver speed of $v=60 \mathrm{~km} / \mathrm{h}$ corresponds to a Doppler frequency equal to $133 \mathrm{~Hz}$. The Doppler spectrum is Jake's and the power loss and delay profiles are: $[0,-1,-3,-9][\mathrm{dB}]$ and $[0$, $1,2,3][\mu s]$ which correspond to a urban type of scenario. Block fading model is assumed, i.e. channels are considered to remain constant during the OFDM block time. No antenna correlation is considered neither at the transmitter nor at the receiver side, which means the four channel impulse responses $\mathbf{h}_{11}, \mathbf{h}_{21}, \mathbf{h}_{12}$ and $\mathbf{h}_{22}$ fade independently. The MMSE equalizer (23) is used to decode the symbols. The four frequency offsets are varying independently in time.

Tracking capability of the proposed method is first demonstrated as a function of time. Real and imaginary parts of $\mathbf{h}_{11}$ are plotted in Figure 3, in the case of $60 \mathrm{~km} / \mathrm{h}$ velocity of the mobile terminal and at $15 \mathrm{~dB}$ SNR. Time variations of the four channel taps are accurately tracked, even for those with low average power. Retraining symbols are sent periodically (every 50-th block in this simulation) in order to avoid loosing the track, which could occur if all the channels in MIMO system happen to fall in a deep fade simultaneously. No special block structure has been considered for retraining, assuming that the whole OFDM symbol is known to the receiver. Retraining time instances are indicated with dotted vertical lines. Decision-directed mode enables the use of limited amount of pilot information: retraining 

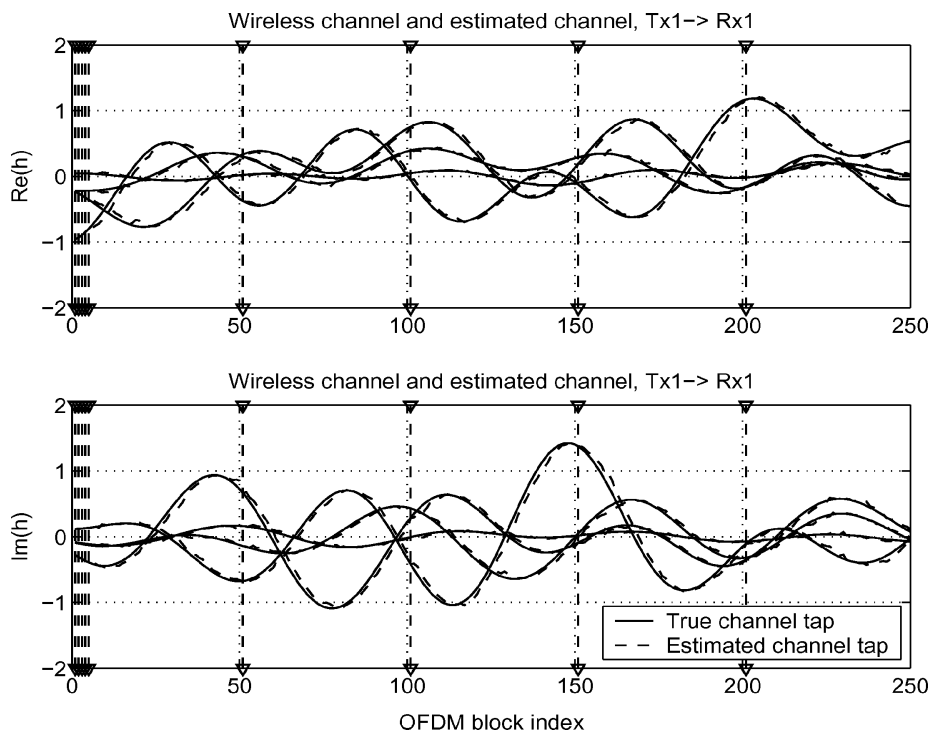

Figure 3. Time-domain tracking of channel coefficients ( $\mathrm{SNR}=15 \mathrm{~dB}, v=60 \mathrm{~km} / \mathrm{h}$ ); vertical lines correspond to retraining time instances.
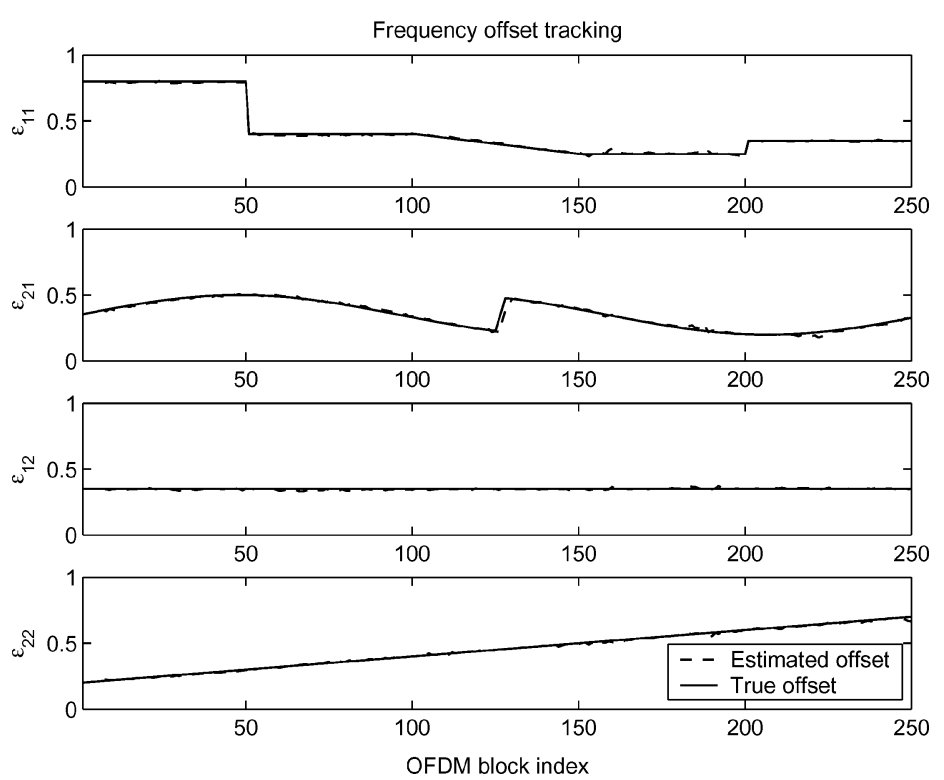

Figure 4. Time-domain tracking of frequency offsets (SNR $=15 \mathrm{~dB}, v=60 \mathrm{~km} / \mathrm{h})$.

every 50 blocks is equivalent to $2 \%$ pilot data rate, compared to the $15-25 \%$ commonly used in mobile wireless applications.

The tracking results for each offset value are shown in Figure 4. In order to demonstrate the tracking capability of our algorithm, each offset varies in time individually, according to a given profile. We note the tracking ability in case of both rapid and slow changes of the time-varying offsets. However, tracking time-varying offsets in OFDM is a sensitive issue when high order modulations are employed [9]. 


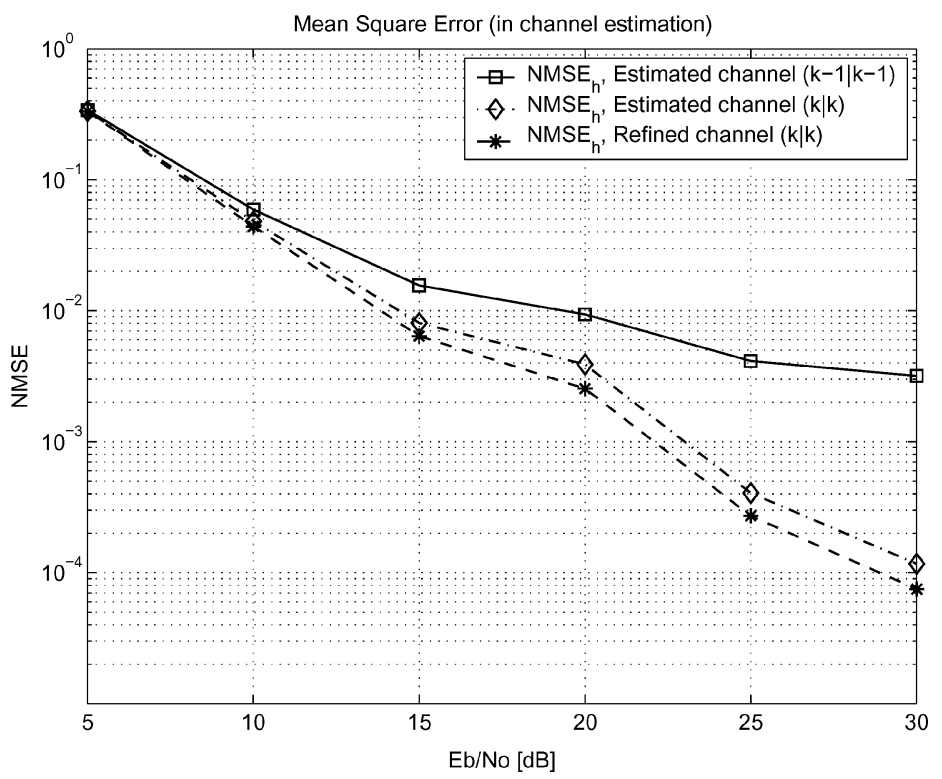

Figure 5. NMSE for channel estimation $(v=30 \mathrm{~km} / \mathrm{h})$.

As a measure of performance of the time-domain estimation, NMSE is computed for both channel and frequency offset as follows:

$$
\begin{aligned}
\operatorname{NMSE}_{\hat{\mathbf{h}}} & =\frac{E\|\hat{\mathbf{h}}-\mathbf{h}\|^{2}}{E\|\mathbf{h}\|^{2}} \\
\mathrm{NMSE}_{\hat{\epsilon}} & =\frac{E\|\hat{\boldsymbol{\epsilon}}-\boldsymbol{\epsilon}\|^{2}}{E\|\boldsymbol{\epsilon}\|^{2}} .
\end{aligned}
$$

The NMSE as a function of SNR is depicted in the case of channel estimation in Figure 5 and for frequency offset estimation in Figure 6. Accurate estimation is obtained over the whole range of SNR. Improved performance is obtained by re-decoding the symbols with the filtered estimates $\hat{\boldsymbol{\epsilon}}(k \mid k)$ and $\hat{\mathbf{h}}(k \mid k)$ and re-running the EKF update stage one more time.

Since the equalization stage operates in the frequency domain, accuracy in estimating frequency responses of the channels at the subcarrier frequencies needs to be investigated. Figures 7 and 8 respectively show amplitude and phase responses, for the true and estimated channels, at a given OFDM block time. Since the time-domain estimation performs well, channel transfer functions are consequently also modeled accurately, both in amplitude and phase. Refined estimates yield frequency responses closer to the true ones. Hence, quality of the subsequent equalization will improve.

Kalman filter has been applied to frequency-domain channel tracking in OFDM [2]. The performance of the proposed method is compared to the frequency domain approach in [2] (no AR modeling included in our simulations) in a SISO case. Time-domain tracking performs well for both amplitude (Figure 9) and phase (Figure 10). The frequency domain approach however looses the track of the phase on some sub-carriers. Tracking in time turns out to be more robust to estimation errors because the frequency correlation of the taps can be efficiently exploited. Furthermore, estimation errors are spread over the whole transmission spectrum, and not concentrated on a given set of subcarriers. The frequency domain method without AR 


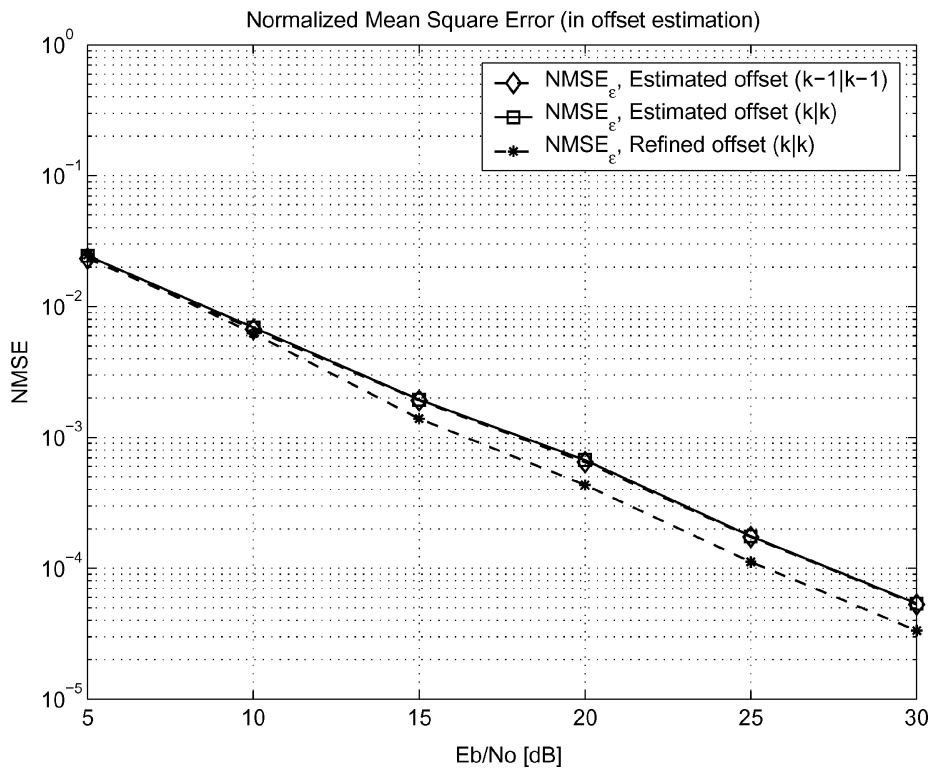

Figure 6. NMSE for frequency offset estimation $(v=30 \mathrm{~km} / \mathrm{h})$.
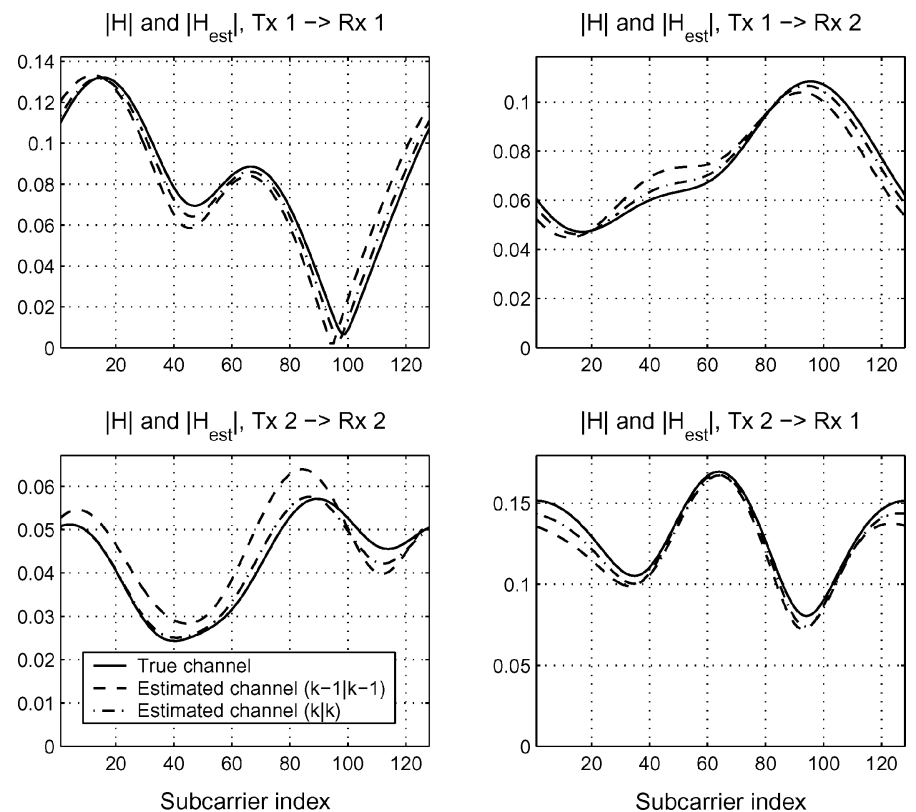

Figure 7. Amplitude response $(\mathrm{SNR}=15 \mathrm{~dB}, v=60 \mathrm{~km} / \mathrm{h})$.

modeling tracks each subcarrier independently. As a result, a single decision error induces an erroneous feedback, provoking a phase rotation for the estimated frequency channel tap (peaks in Figure 10). This results in a complete loss of the data stream on the corresponding subcarrier.

Simulations for different terminal velocities ranging from $3 \mathrm{~km} / \mathrm{h}$ (pedestrian mobility) up to $140 \mathrm{~km} / \mathrm{h}$ have been performed at $20 \mathrm{~dB}$ SNR, see Figure 11. As expected, the performance degrades as the speed increases, due to cumulating decision errors since the proposed algorithm 
$\arg (\mathrm{H})$ and $\arg \left(\mathrm{H}_{\mathrm{est}}\right)$, Tx $1 \rightarrow \mathrm{Rx} 1$

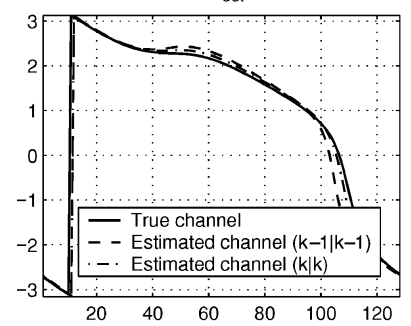

$\arg (\mathrm{H})$ and $\arg \left(\mathrm{H}_{\text {est }}\right), \mathrm{T} \times 2 \rightarrow \mathrm{Rx} 2$

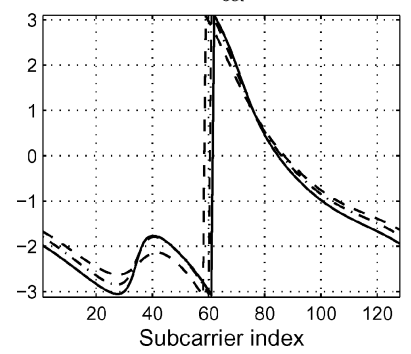

$\arg (\mathrm{H})$ and $\arg \left(\mathrm{H}_{\text {est }}\right), \mathrm{T} \times 1 \rightarrow \mathrm{Rx} 2$

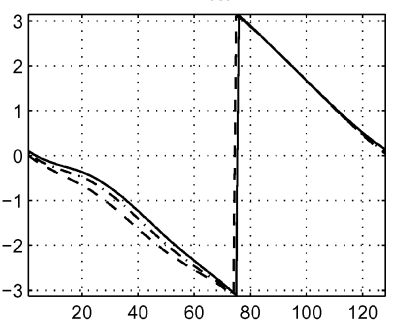

$\arg (\mathrm{H})$ and $\arg \left(\mathrm{H}_{\text {est }}\right)$, Tx $2 \rightarrow \mathrm{Rx} 1$

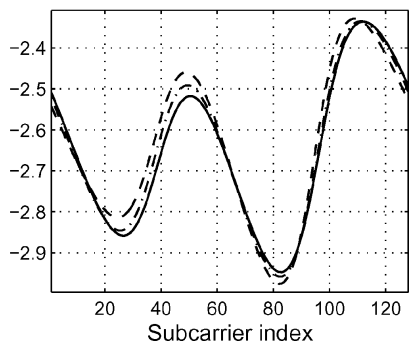

Figure 8 . Phase response $(\mathrm{SNR}=15 \mathrm{~dB}, v=60 \mathrm{~km} / \mathrm{h})$.

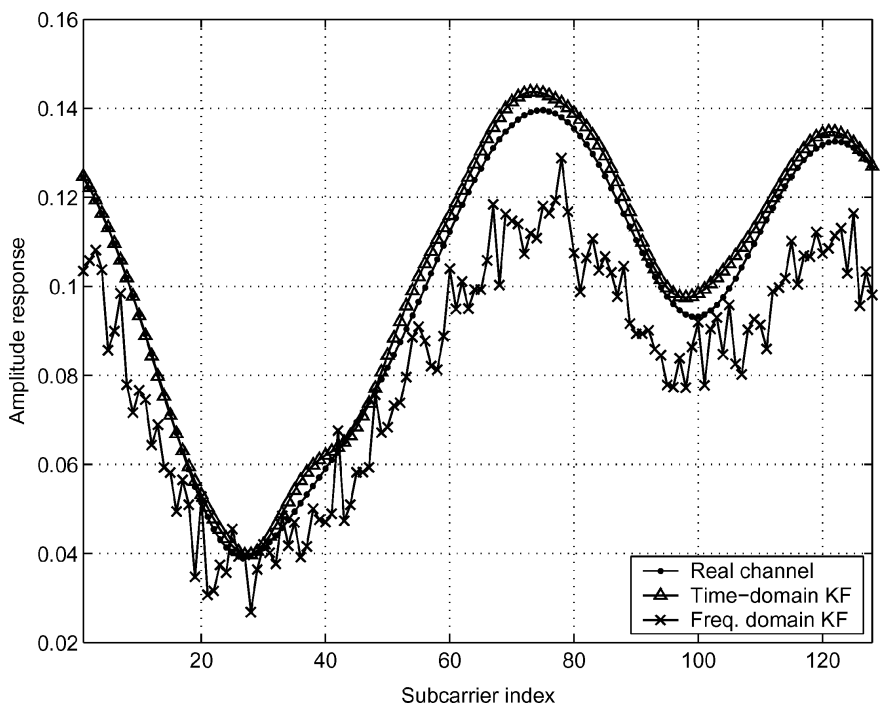

Figure 9. Amplitude response ( $\mathrm{SNR}=15 \mathrm{~dB}, v=30 \mathrm{~km} / \mathrm{h})$.

is working in a decision-directed mode. Retraining has been used every 50-th symbol block. High terminal velocities translate into high time-selectivity of the wireless channel. Hence, the channel may have changed and the previous estimate may not be adequate to make correct symbol decisions and keep the track of channel coefficients. A well-designed pilot structure in time-frequency domains is required to have reliable tracking performance at high velocities $[8,10,32]$. For medium speeds we observe an improvement in BER when using the filtered channel estimate at time $k$. This requires that symbols are decoded twice. From our simulations experiments we have observed that further iteration (refinement) of EKF did not improve significantly the performance. 


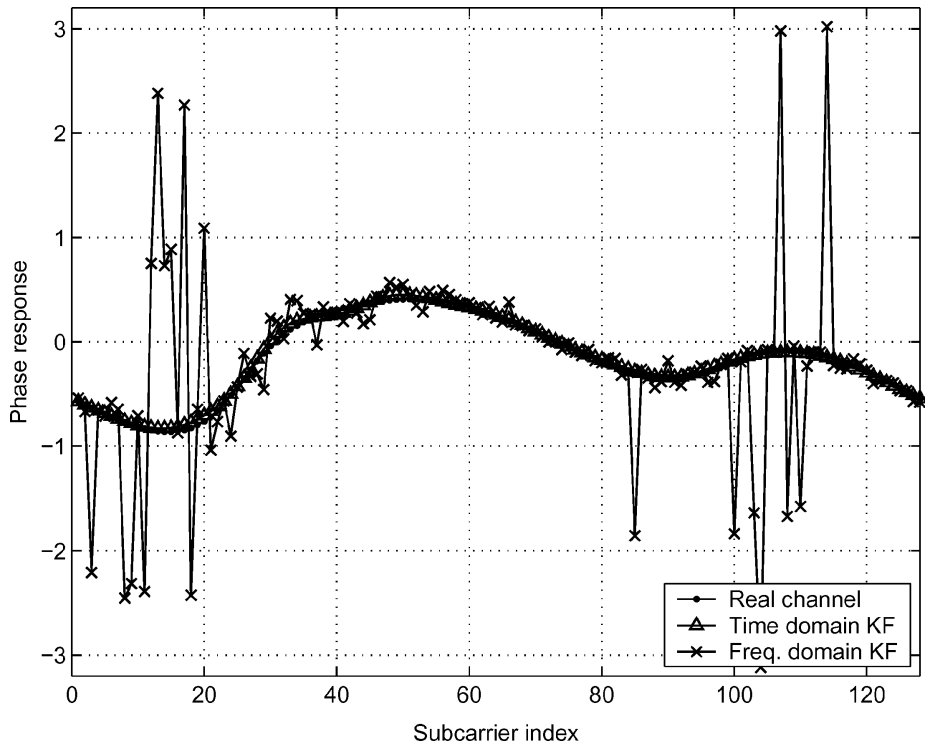

Figure 10. Phase response $(\mathrm{SNR}=15 \mathrm{~dB}, v=30 \mathrm{~km} / \mathrm{h})$.

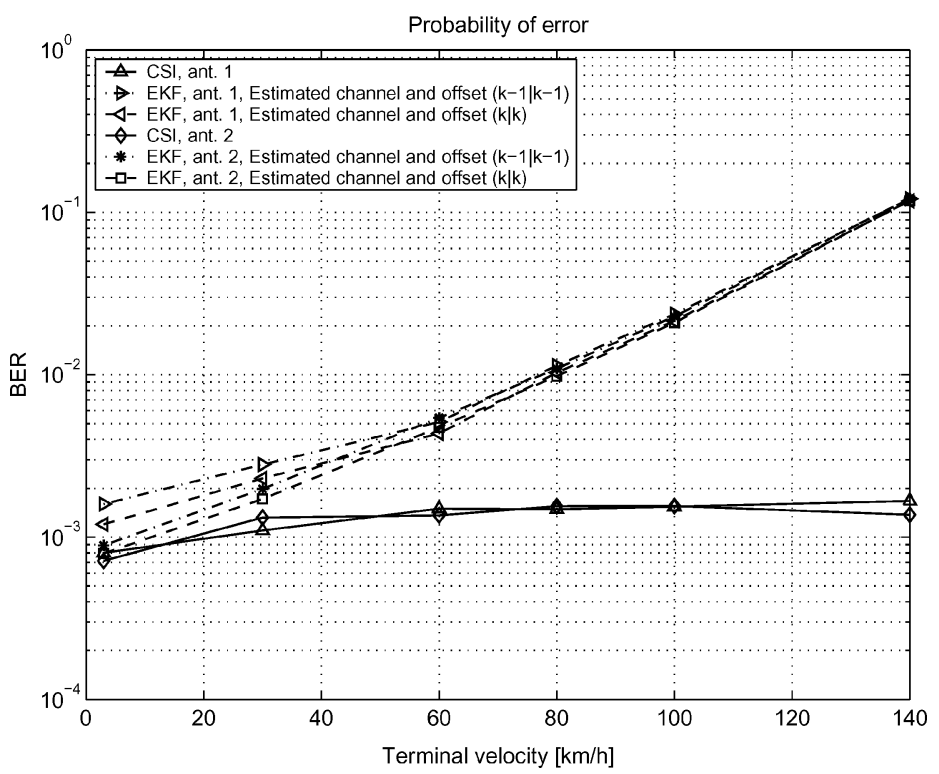

Figure 11. Bit error rate performance for variable terminal velocity, decision-directed mode with no pilot signal $(\mathrm{SNR}=20 \mathrm{~dB})$.

Finally, the bit error rate is considered as a function of noise variance. The results are presented in Figure 12 for a terminal velocity set to $30 \mathrm{~km} / \mathrm{h}$. A lower bound for the performance of the tracking algorithm is given by using the ideal channel state information (CSI), i.e. perfectly known channels and frequency offsets at the receiver side. Simulation results highlight the good performance of the time-domain tracking algorithm for a wide range of SNR. Lower terminal speeds lead to lower error rates, close to the ones obtained with a perfectly known channel. In this simulation both the channel and the frequency offsets have been estimated and tracked. We emphasize the fact that, with modeling one offset per MIMO branch, MMSE 


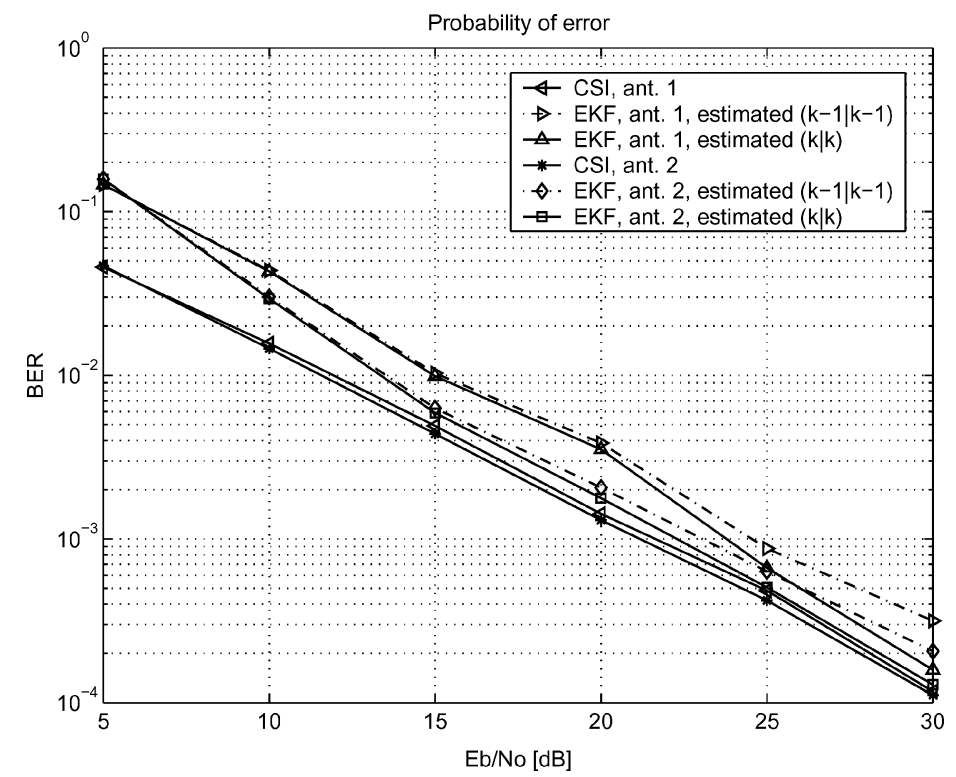

Figure 12. Bit error rate performance (over 1000 blocks, $v=30 \mathrm{~km} / \mathrm{h}$ ), at receive antennas 1 and 2.

frequency domain equalization based on the filtered channel and offset estimates from previous time instance $(k-1)$ may not be sufficiently accurate to reliably decode the symbols, especially for high order modulations. However enhanced equalizers might be needed to fully compensate for the induced intercarrier interference [33].

\section{Conclusions}

In this paper, a channel and frequency offset estimation and tracking method stemming from extended Kalman filter is proposed for MIMO OFDM based systems. Each MIMO channel branch is considered to introduce its own frequency offset, which is caused by separate RF-IF chains in each transmitter and receiver as well as the rich scattering environment and the large angle spread which are desired in MIMO scenarios. The tracking stage runs in time-domain and is the core of the presented algorithm. Reliable offset tracking capability is also shown in cases when the frequency offset varies rapidly in time. Moreover, very little or no pilot data were needed, except during the initialization stage. The benefits of the time-domain approach are twofold: first superior tracking performance and robustness are achieved compared to solutions purely working in the frequency domain. Second, computational complexity is significantly lowered as well. However, in more demanding environments characterized by low SNR and fast varying channels, additional pilot symbols may be needed to avoid the divergence of the algorithm. The reliable performance of the method is demonstrated for mobile user in a typical urban scenario for different velocities.

\section{References}

1. R. van Nee and R. Prasad, "OFDM for Wireless Multimedia Communications”, Artech House, p. 260, 2000.

2. S.B. Bulumulla, S.A. Kassam and S.S. Venkatesh, "An Adaptive Diversity Receiver for OFDM in Fading Channels", in Proceedings of International Conference on Communications, Vol. 3, pp. 1325-1329, 1998. 
3. J.-J. van de Beek, O. Edfors, M. Sandell, S.K. Wilson and P.O. Borjesson, "On channel estimation in OFDM systems", in Proceedings of 45th IEEE Vehicular Technology Conference, pp. 815-819, 1995.

4. O. Edfors, M. Sandell, J.-J. van de Beek, S.K. Wilson and P.O. Borjesson, "OFDM Channel Estimation by Singular Value Decomposition”, IEEE Transactions on Communications, Vol. 46, pp. 931-939, 1998.

5. Y.G. Li, J.H. Winters and N.R. Sollenberger, "MIMO-OFDM for Wireless Communications: Signal Detection with Enhanced Channel Estimation", IEEE Transactions on Communications, Vol. 50, No. 9, pp. 1471-1477, 2002.

6. Y.G. Li, "Simplified Channel Estimation for OFDM Systems with Multiple Transmit Antennas", IEEE Transactions on Wireless Communications, Vol. 1, pp. 67-75, 2002.

7. Y.G. Li, N. Seshadri and S. Ariyavisitakul, "Channel Estimation for OFDM Systems with Transmitter Diversity in Mobile Wireless Channels", IEEE Journal on Selected Areas in Communications, Vol. 17, pp. 461-471, 1999.

8. I. Barhumi, G. Leus and M. Moonen, "Optimal Training Design for MIMO OFDM Systems in Mobile Wireless Channels", IEEE Transactions on Signal Processing, Vol. 51, No. 6, pp. 1615-1624, 2003.

9. H. Nishookar and R. Prasad, "On the Sensitivity of Multicarrier Transmission over Multipath Channels to Phase Noise and Frequency Offset", in Proceedings of IEEE International Symposium on Personal, Indoor and Mobile Radio Communications, PIMRC, Vol. 1, pp. 68-72, 1996.

10. S. Coleri, M. Ergen, A. Puri and A. Bahai, "Channel Estimation Techniques Based on Pilot Arrangement in OFDM Systems", IEEE Transactions on Broadcasting, Vol. 48, No. 3, pp. 223-229, 2002.

11. B.-S. Chen and C.-L. Tsai, "Frequency Offset Estimation in an OFDM System", in IEEE Workshop on Signal Processing Advances in Wireless Communications, pp. 150-153, 2001.

12. S.B. Bulumulla, S.A. Kassam and S.S. Venkatesh, "A Systematic Approach to Detecting OFDM Signals in a Fading Channel”, IEEE Transactions on Communications, Vol. 48, No. 5, pp. 725-728, 2000.

13. T. Roman, M. Enescu and V. Koivunen, "Time-Domain Method for Tracking Dispersive Channels in MIMO OFDM Systems", in Proceedings of IEEE Conference on Acoustics, Speech, and Signal Processing, ICASSP, Vol. 4, pp. 393-396, 2003

14. C. Zhenlan and D. Dahlhaus, "Time Versus Frequency Domain channel Tracking Using Kalman Filters for OFDM Systems with Antenna Arrays", in Proceedings of the IEEE Vehicular Technology Conference, VTC 2003-Spring, Vol. 1, pp. 651-655, 2003.

15. R. Perets and B. Bobrovsky, "A New Phase and Frequency Offset Estimation Algorithm for OFDM Systems Applying Kalman Filter", in The 22nd Convention of Electrical and Electronics Engineers in Israel, pp. 300302, 2002.

16. R.A. Iltis, "Joint Estimation of pn Code Delay and Multipath Using the Extended Kalman Filter", IEEE Transactions on Communications, Vol. 38, No. 10, pp. 1677-1685, 1990.

17. T. Roman, M. Enescu and V. Koivunen, "Joint Time-Domain Method Tracking of Channel and Frequency Offset for OFDM Systems", in Proceedings of IEEE Workshop on Signal Processing Advances in Wireless Communications, SPAWC, 2003.

18. T. Roman, M. Enescu and V. Koivunen, "Recursive Estimation of Time-Varying Channel and Frequency Offset in MIMO OFDM Systems", in Proceedings of the IEEE Conference on Personal, Indoor and Mobile Radio Communications, PIMRC, Vol. 2, pp. 1934-1938, 2003.

19. O. Besson and P. Stoica, "On Parameter Estimation of MIMO Flat-Fading Channels with Frequency Offsets", IEEE Transactions on Signal Processing, Vol. 51, No. 3, pp. 602-613, 2003.

20. Z. Wang and G.B. Giannakis, "Wireless Multicarrier communications", IEEE Signal Processing Magazine, Vol. 17, No. 3, pp. 29-48, 2000.

21. Z. Liu, G.B. Giannakis and B.L. Hughes, "Double Differential Spacetime Block coding for TimeSelective Fading Channels", IEEE Transactions on Communications, Vol. 49, No. 9, pp. 1529-1539, 2001.

22. A. Jakobsson, A.L. Swindlehurst and P. Stoica, "Resolution of Overlapping Doppler Shifted Echoes", in Proceedings of the IEEE International Conference on Acoustics, Speech, and Signal Processing, Vol. 4, pp. 2417-2420, 1998.

23. A. Jakobsson, A.L. Swindlehurst and P. Stoica, "Subspace-Based Estimation of Time Delays and Doppler Shifts", IEEE Transactions on Signal Processing, Vol. 46, No. 9, pp. 2472-2482, 1998.

24. H.A. Cirpan and M.K. Tsatsanis, "Maximum Likelihood Blind Channel Estimation in the Presence of Doppler Shifts", IEEE Transactions on Signal Processing, Vol. 47, No. 6, pp. 1559-1569, 1999. 


\section{T. Roman et al.}

25. A.W. Habboosh, R.J. Vaccaro and S. Kay, "An Algorithm for Detecting Closely Spaced Delay/Doppler Components", in Proceedings of the IEEE International Conference on Acoustics, Speech, and Signal Processing, Vol. 1, pp. 535-538, 1997.

26. P. Stoica and G. Ganesan, "Trained Space-Time Block Decoding for Flat Fading Channels with Frequency Offsets", Kluwer Wireless Personal Communications, No. 27, pp. 235-245, 2003.

27. G. Giannakis, Y. Hua, P. Stoica and L. Tong (eds.), Signal Processing Advances in Wireless and Mobile Communications, Volume 1: Trends in Channel Estimation and Equalization. Prentice Hall, 2000.

28. G. Leus and M. Moonen, "Per-Tone Equalization for MIMO OFDM Systems", IEEE Transactions on Signal Processing, Vol. 51, No. 11, pp. 2965-2975, 2003.

29. M. Tsatsanis, G. Giannakis and G. Zhou, "Estimation and Equalization of Fading Channels with Random Coefficients", Signal Processing, Vol. 53, No. 2-3, pp. 211-229, 1996.

30. C. Komninakis, C. Fragouli, A.H. Sayed and R. Wesel, "Multi-Input Multi-Output Fading Channel Tracking and Equalization Using Kalman Estimation”, IEEE Transactions on Signal Processing, Vol. 50, No. 5, pp. 1065-1076, 2002.

31. M. Enescu and V. Koivunen, "On the Estimation of State Transition Matrix and Noise Statistics in State-Space Models", in Proceedings of the IEEE Vehicular Technology Conference, VTC 2002-Fall, Vol. 4, pp. 2192-2196, 2002.

32. R. Negi and J. Cio., "Pilot Tone Selection for Channel Estimation in a Mobile OFDM System", IEEE Transactions for Consumer Electronics, Vol. 44, No. 3, pp. 1122-1128, 1998.

33. A. Stamoulis, S.N. Diggavi and N. Al-Dhahir, "Intercarrier Interference in MIMO OFDM", IEEE Transactions on Signal Processing, Vol. 50, No. 10, pp. 2451-2463, 2002. 